\title{
ON THE CURVATURE OF THE INDICATRIX SURFACE IN THREE-DIMENSIONAL MINKOWSKI SPACES
}

\author{
CS.VINCZE
}

\begin{abstract}
As it is well-known a Minkowski space is a finite dimensional real vector space equipped with a Minkowski functional $F$. By the help of its second order partial derivatives we can introduce a Riemannian metric on the vector space and the indicatrix hypersurface $S:=F^{-1}(1)$ can be can be investigated as a Riemannian submanifold in the usual sense. If the Minkowski functional is quadratic then we have an Euclidean space and the indicatrix has constant 1 curvature. In his classical paper [1] F. Brickell proved that the converse is also true provided that the indicatrix is symmetric with respect to the origin. M. Ji and Z. Shen investigated the (sectional) curvature of Randers indicatrices and it always turned out greater than zero and less or equal than 1 ; see [3]. In this note we give a general lower and upper bound for the curvature in terms of the norm of the Cartan tensor.
\end{abstract}

\section{Preliminaries}

1.1. Minkowski functionals. Let $V$ be an $n$-dimensional $(n \geq 2)$ real vector space. The elements of $V$ will be interpreted both as points $p, q, \ldots$ and vectors $v, w, \ldots$ as usual. A Minkowski functional on $V$ is a function $F: V \rightarrow \mathbb{R}$ with the following properties:

(F0) $\forall p \in V \backslash\{0\}: F(p)>0$ and $F(0)=0$.

(F1) $F$ is positive homogeneous of degree 1, i.e. $\forall t \in \mathbb{R}^{+}: F(t p)=t F(p)$.

(F2) $F$ is continuous on $V$ and smooth over the set $V \backslash\{0\}$.

(F3) $\forall p \in V \backslash\{0\}$ :

$$
g_{p}:=E^{\prime \prime}(p): V \times V \rightarrow \mathbb{R}
$$

is an inner product on $V$, where $E:=\frac{1}{2} F^{2}$ is the energy function.

By the homogeneity condition (F1) we have

$$
g_{p}(p, v)=E^{\prime}(p)(v) \text { and } g_{p}(p, p)=2 E(p) .
$$

1.2. Cartan tensors. Let $(V, F)$ be a Minkowski space and consider the mappings

$$
\mathcal{C}_{b}(p):=E^{\prime \prime \prime}(p): V \times V \times V \rightarrow \mathbb{R} \text { and } \mathcal{C}_{p}: V \times V \rightarrow V
$$

defined by the formula

$$
g_{p}\left(\mathcal{C}_{p}(v, w), z\right)=\mathcal{C}_{b}(p)(v, w, z) .
$$

The mapping $\mathcal{C}$ is called the first Cartan tensor while its lowered tensor is the second Cartan tensor. They are totally symmetric and, of course, multilinear. This means that the mapping

$$
\mathcal{C}_{p}(v, \cdot): V \rightarrow V, \quad \mathcal{C}_{p}(v, \cdot)(w):=\mathcal{C}_{p}(v, w)
$$


is a self-adjoint linear operator with respect to the inner product $g_{p}$. Using the homogeneity condition (F1) again it follows that

$$
\mathcal{C}_{p}(p, \cdot)=0
$$

We set

$$
\|\mathcal{C}\|_{p}:=\max _{v \in S_{p}} \mathcal{C}_{b}(v, v, v),
$$

where $S_{p}:=\left\{v \in V \mid g_{p}(v, v)=1\right\}$. Consider now the indicatrix hypersurface $S:=F^{-1}(1)$ of the Minkowski space and let us form the norm of the lowered Cartan tensor as follows

$$
\|\mathcal{C}\|:=\max _{p \in S}\|\mathcal{C}\|_{p}
$$

It is well-known that the vanishing of the first or, in an equivalent way, the second Cartan tensor implies the Minkowski space to be Euclidean.

Remark 1. In what follows we shall suppose that $\operatorname{dim} V \geq 3$ without any further comment.

1.3. Riemannian quantities. According to the regularity property (F3), any Minkowski space can be considered as a Riemannian manifold in the usual sense. After identifying the tangent spaces with $V$, consider the following special vector fields:

$$
\begin{aligned}
& X: V \rightarrow V, p \longrightarrow X_{p}:=x, \\
& Y: V \rightarrow V, \quad p \longrightarrow Y_{p}:=y, \\
& C: V \rightarrow V, \quad p \longrightarrow C_{p}:=p
\end{aligned}
$$

where $x, y$ in $V$ are arbitrarily fixed vectors and $C$ is the so-called Liouville vector field. It can be easily seen that the Lévi-Civita connection $\nabla$ associated with $g$ acts as follows:

$$
\nabla_{X_{p}} Y=\mathcal{C}_{p}(x, y)
$$

and, consequently, the curvature tensor has the following simple form:

$$
R_{p}(x, y) z=\mathcal{C}_{p}\left(\mathcal{C}_{p}(x, z), y\right)-\mathcal{C}_{p}\left(x, \mathcal{C}_{p}(y, z)\right) .
$$

According to the property (4) both the first and the second Cartan tensor vanishes along the Liouville vector field and thus, for any tangent vector $X_{p} \in V:$

$$
\nabla_{X_{p}} C=X_{p}
$$

On the other hand, by the equation (1), the Liouville vector field is just a normal unit vector field of the indicatrix hypersurface $S$. This means that $S$ is a totally umbilical hypersurface with respect to the Riemannian metric $g$. More precisely, for any point $p \in S$ the shape operator $\eta_{p}$ acts as follows:

$$
\eta_{p}: T_{p} S \rightarrow T_{p} S, \quad X_{p} \longrightarrow \eta_{p}\left(X_{p}\right):=-\nabla_{X_{p}} C=-X_{p}
$$


and, consequently, the sectional curvature $\kappa_{p}(x, y)$ can be calculated by the following formula:

$$
\begin{aligned}
\kappa_{p}(x, y) & =g_{p}\left(R_{p}(x, y) y, x\right)+\operatorname{det}\left(\begin{array}{ll}
g_{p}\left(\eta_{p}(x), x\right) & g_{p}\left(\eta_{p}(x), y\right) \\
g_{p}\left(\eta_{p}(y), x\right) & g_{p}\left(\eta_{p}(y), y\right)
\end{array}\right)= \\
& =g_{p}\left(R_{p}(x, y) y, x\right)+1= \\
& \stackrel{(8)}{=} g_{p}\left(\mathcal{C}_{p}(x, y), \mathcal{C}_{p}(x, y)\right)-g_{p}\left(\mathcal{C}_{p}(x, x), \mathcal{C}_{p}(y, y)\right)+1,
\end{aligned}
$$

where $x, y \in T_{p} S$ are orthonormal tangent vectors. In this note we are going to prove the following main result:

Suppose that $\operatorname{dim} V=3$; then for any $p \in S$ the curvature $\kappa_{p}$ of the indicatrix surface satisfies the following relations:

$$
1-\frac{\|\mathcal{C}\|_{p}^{2}}{4} \leq \kappa_{p} \leq 1-2\|\mathcal{C}\|_{p}^{2}
$$

and, consequently,

$$
1-\frac{\|\mathcal{C}\|^{2}}{4} \leq \kappa \leq 1-2\|\mathcal{C}\|^{2} .
$$

\section{The PROOF OF THE MAIN THEOREM}

Lemma 1. Let us choose a vector $x_{0} \in S_{p}$ such that

$$
\|\mathcal{C}\|_{p}=\mathcal{C}_{b}(p)\left(x_{0}, x_{0}, x_{0}\right) .
$$

If the vector $y_{0} \in S_{p}$ is orthogonal to $x_{0}$, i.e. $g_{p}\left(x_{0}, y_{0}\right)=0$, then

$$
g_{p}\left(\mathcal{C}_{p}\left(x_{0}, x_{0}\right), y_{0}\right)=0
$$

and the following estimation holds:

$$
-\|\mathcal{C}\|_{p} \leq g_{p}\left(\mathcal{C}_{p}\left(x_{0}, y_{0}\right), y_{0}\right) \leq \frac{1}{2}\|\mathcal{C}\|_{p} .
$$

Proof. Let us form the function

$$
\begin{aligned}
f(t) & :=g_{p}\left(\mathcal{C}_{p}\left(\cos t x_{0}+\sin t y_{0}, \cos t x_{0}+\sin t y_{0}\right), \cos t x_{0}+\sin t y_{0}\right)= \\
& =\cos ^{3} t g_{p}\left(\mathcal{C}_{p}\left(x_{0}, x_{0}\right), x_{0}\right)+3 \cos ^{2} t \sin t g_{p}\left(\mathcal{C}_{p}\left(x_{0}, x_{0}\right), y_{0}\right)+ \\
& +3 \cos t \sin ^{2} t g_{p}\left(\mathcal{C}_{p}\left(x_{0}, y_{0}\right), y_{0}\right)+\sin ^{3} t g_{p}\left(\mathcal{C}_{p}\left(y_{0}, y_{0}\right), y_{0}\right) .
\end{aligned}
$$

Since $f$ attains its maximum at the point $t=0$, we have that $f^{\prime}(0)=0$ and, of course, $f^{\prime \prime}(0) \leq 0$. On the other hand

$$
\begin{aligned}
& f^{\prime}(0)=3 g_{p}\left(\mathcal{C}_{p}\left(x_{0}, x_{0}\right), x_{0}\right), \\
& f^{\prime \prime}(0)=-3\left(g_{p}\left(\mathcal{C}_{p}\left(x_{0}, x_{0}\right), x_{0}\right)-2 g_{p}\left(\mathcal{C}_{p}\left(x_{0}, y_{0}\right), y_{0}\right)\right)
\end{aligned}
$$

which means that (14) and the upper bound of the estimation (15) are valid. According to the equation (14) the expression of the function $f$ can be reduced to the following simple form:

$$
\begin{aligned}
f(t) & =\cos ^{3} t g_{p}\left(\mathcal{C}_{p}\left(x_{0}, x_{0}\right), x_{0}\right)+3 \cos t \sin ^{2} t g_{p}\left(\mathcal{C}_{p}\left(x_{0}, y_{0}\right), y_{0}\right)+ \\
& +\sin ^{3} t g_{p}\left(\mathcal{C}_{p}\left(y_{0}, y_{0}\right), y_{0}\right) .
\end{aligned}
$$

Consider now the auxiliary function

$$
h:\left(0, \frac{\pi}{2}\right) \rightarrow \mathbb{R}, \quad t \longrightarrow h(t):=f(t)+f(-t) .
$$


It is clear from the definition that for any $t \in \mathbb{R}: f(t) \geq-\|\mathcal{C}\|_{p}$ and, consequently,

$$
h(t) \geq-2\|\mathcal{C}\|_{p} \Rightarrow g_{p}\left(\mathcal{C}_{p}\left(x_{0}, x_{0}\right), y_{0}\right) \geq-\frac{1+\cos ^{3} t}{3 \cos t \sin t}\|\mathcal{C}\|_{p}
$$

Since the function

$$
t \longrightarrow \frac{1+\cos ^{3} t}{3 \cos t \sin t} \quad\left(0 \leq t \leq \frac{\pi}{2}\right)
$$

attains its global minimum at the point $t=\frac{\pi}{3}$, we have the lower bound of the estimation (15).

Lemma 2. Keeping the conditions of Lemma 1 we have that

$$
\mathcal{C}_{p}\left(x_{0}, x_{0}\right)=\|\mathcal{C}\|_{p} x_{0}
$$

i.e. $x_{0}$ is the eigenvector of the mapping $\mathcal{C}_{p}\left(x_{0}, \cdot\right)$ and the corresponding eigenvalue is just the norm of the lowered Cartan tensor at the point $p$.

Proof. Without loss of generality we may suppose that $x_{0}$ is tangential to the indicatrix hypersurface, i.e. $g_{p}\left(p, x_{0}\right)=0$. Indeed, in case of $\|\mathcal{C}\|_{p}=0$ it is trivial. According to the property (4), if $\|\mathcal{C}\|_{p}>0$, then, of course, $x_{0}$ must be tangential to the indicatrix surface. In order to see this observation let us form the tangential component

$$
x_{0}^{\top}:=x_{0}-\frac{1}{F^{2}(p)} g_{p}\left(p, x_{0}\right) \Rightarrow g_{p}\left(x_{0}^{\top}, x_{0}^{\top}\right)=1-\frac{1}{F^{2}(p)} g_{p}^{2}\left(p, x_{0}\right) .
$$

Then, as an easy calculation shows,

$$
\|\mathcal{C}\|_{p}=\mathcal{C}_{b}(p)\left(x_{0}, x_{0}, x_{0}\right) \stackrel{(4)}{=} \mathcal{C}_{b}(p)\left(x_{0}^{\top}, x_{0}^{\top}, x_{0}^{\top}\right) \leq\left(1-\frac{1}{F^{2}(p)} g_{p}^{2}\left(p, x_{0}\right)\right)^{\frac{3}{2}}\|\mathcal{C}\|_{p},
$$

which is obviously a contradiction unless $g_{p}\left(p, x_{0}\right)=0$, as was to be observed. Let now $x_{0}, y_{0}, \ldots, \frac{1}{F(p)} p$ be an orthonormal basis at the point $p$. Then

$$
\begin{array}{r}
\mathcal{C}_{p}\left(x_{0}, x_{0}\right)=g_{p}\left(\mathcal{C}_{p}\left(x_{0}, x_{0}\right), x_{0}\right) x_{0}+g_{p}\left(\mathcal{C}_{p}\left(x_{0}, x_{0}\right), y_{0}\right) y_{0}+\ldots \\
\ldots+\frac{1}{F^{2}(p)} g_{p}\left(\mathcal{C}_{p}\left(x_{0}, x_{0}\right), p\right) p=g_{p}\left(\mathcal{C}_{p}\left(x_{0}, x_{0}\right), x_{0}\right) x_{0}
\end{array}
$$

using Lemma 1 and (4).

Theorem 1. Suppose that $\operatorname{dim} V=3$; then for any $p \in S$ the curvature $\kappa_{p}$ of the indicatrix surface satisfies the following relations:

$$
1-\frac{\|\mathcal{C}\|_{p}^{2}}{4} \leq \kappa_{p} \leq 1-2\|\mathcal{C}\|_{p}^{2}
$$

and, consequently,

$$
1-\frac{\|\mathcal{C}\|^{2}}{4} \leq \kappa \leq 1-2\|\mathcal{C}\|^{2} .
$$

Proof. Consider a vector $x_{0} \in S_{p}$ such that

$$
\|\mathcal{C}\|_{p}=\mathcal{C}_{b}(p)\left(x_{0}, x_{0}, x_{0}\right) ;
$$

then, as we have seen, $x_{0}$ is an eigenvector of the mapping $\mathcal{C}_{p}\left(x_{0}, q c d o t\right)$. Since it is a self-adjoint operator, there exists a $g_{p}$-orthonormal system $\left(x_{0}, y_{0}, p\right)$ of eigenvectors, where $x_{0}$ and $y_{0}$ are tangential to the indicatrix surface ( $p$ is, of course, a normal vector ) at the point $p$. The system 
$\left(\|\mathcal{C}\|_{p}, \lambda_{p}, 0\right)$ of scalars is the full spectrum; see Lemma 2 and (4). According to the formula (11) it follows that

$$
\kappa_{p}=\lambda_{p}^{2}-\lambda_{p}\|\mathcal{C}\|_{p}+1,
$$

where, by Lemma 1 , the eigenvalue $\lambda_{p}=g_{p}\left(\mathcal{C}_{p}\left(x_{0}, y_{0}\right), y_{0}\right)$ satisfies the relations

$$
-\|\mathcal{C}\|_{p} \leq \lambda_{p} \leq \frac{1}{2}\|\mathcal{C}\|_{p}
$$

Evaluating the function $f(t):=t^{2}-t\|\mathcal{C}\|_{p}+1$ at the endpoints, we have (19) immediately.

\section{Some Remarks on the CURVATURe of the indicAtrix HYPERSURFACE}

3.1. The contracted Cartan tensor. Consider the contracted Cartan tensor

$$
\tilde{\mathcal{C}_{p}}(x):=\operatorname{trace} \mathcal{C}_{p}(x, \cdot)
$$

and let us define a vector field $\theta: V \rightarrow V$ in the following way:

$$
\left.g_{p}\left(\theta_{p}, x\right)=\mathcal{C}_{p} \tilde{(} x\right) .
$$

It is well-known that $\theta$ is just the gradient of the function

$$
f:=\ln \sqrt{\operatorname{det} g_{i j}},
$$

where $g_{i j}(p):=g_{p}\left(e_{i}, e_{j}\right)$ and $\left(e_{1}, \ldots, e_{n}\right)$ is a basis of $V$. Since $f$ is homogeneous of degree zero it attains both a global minimum and maximum on $\mathrm{V}$. This means that there exists at least two different points $p$ and $q$ such that $\theta_{p}=0$ and, consequently,

$$
0=\tilde{\mathcal{C}_{p}}\left(x_{0}\right)=g_{p}\left(\mathcal{C}_{p}\left(x_{0}, x_{0}\right), x_{0}\right)+g_{p}\left(\mathcal{C}_{p}\left(x_{0}, y_{0}\right), y_{0}\right) \Rightarrow \lambda_{p}=-\|\mathcal{C}\|_{p}
$$

under the condition $\operatorname{dim} V=3$. Then, of course,

$$
\kappa_{p}=1+2\|\mathcal{C}\|_{p}^{2} \text {. }
$$

3.2. The area of the indicatrix surface. Using the Gauss-Bonnet theorem it follows that

$$
1-\frac{\|\mathcal{C}\|^{2}}{4} \leq \frac{4 \pi}{A(S)} \leq 1+2\|\mathcal{C}\|^{2}
$$

where $A(S)$ is the area of the indicatrix surface with respect to the induced Riemannian metric.

Remark 2. If the Minkowski functional is reversible, i.e. $F(v)=F(-v)$ then, by the Santalo's inequality, $A(S) \leq 4 \pi$; see e.g. [2] and [4]. This means that the relation on the left hand side is trivial provided that the indicatrix surface is symmetric with respect to the origin. 
3.3. Asanov-type energy functions. Consider the three-dimensional coordinate space $\mathbb{R}^{3}$ equipped with the canonical inner product. The elements of the space will be interpreted in the form

$$
p=q+t \frac{\partial}{\partial t}
$$

where $q \in \mathbb{R}^{2}$ and $t \in \mathbb{R}$. We set

$$
B(p):=\|q\|^{2}+\frac{K}{2}|t|\|q\|+t^{2},
$$

where $-4<K<4$. In his paper [1] G. S. Asanov proved that the lowered Cartan tensor associated with the energy function

$$
E(p):=\frac{1}{2} B(p) \exp \frac{2 K}{\sqrt{16-K^{2}}}\left(\arctan \frac{\sqrt{16-K^{2}}|t|}{K|t|+4\|q\|}-\arctan \frac{\sqrt{16-K^{2}}}{K}\right)
$$

has the following form:

$$
\begin{aligned}
\mathcal{C}_{b}(p)(x, y, z) & =\frac{1}{3}\left(\tilde{\mathcal{C}_{p}}(x) h_{p}(y, z)+\tilde{\mathcal{C}_{p}}(y) h_{p}(x, z)+\tilde{\mathcal{C}_{p}}(z) h_{p}(x, y)\right)- \\
& -\frac{4 E(p)}{K^{2}}\left(\frac{2}{3}\right)^{3} \tilde{\mathcal{C}_{p}}(x) \tilde{\mathcal{C}_{p}}(y) \tilde{\mathcal{C}_{p}}(z)
\end{aligned}
$$

where

$$
h_{p}:=g_{p}-d_{p} F \otimes d_{p} F
$$

is the so-called angular metric tensor. In a standard terminology, the Minkowski space $\left(\mathbb{R}^{3}, E\right)$ is semi-C-reducible; for the details see [1] and [2]. Under the conditions of Lemma 1

$$
0=\mathcal{C}_{b}(p)\left(x_{0}, x_{0}, y_{0}\right) \stackrel{(29)}{=} \frac{1}{3} \tilde{\mathcal{C}_{p}}\left(y_{0}\right)-\frac{2}{K^{2}}\left(\frac{2}{3}\right)^{3} \tilde{\mathcal{C}}_{p}^{2}\left(x_{0}\right) \tilde{\mathcal{C}_{p}}\left(y_{0}\right)
$$

provided that $p \in S$, i.e. $E(p)=\frac{1}{2}$. If $\tilde{\mathcal{C}_{p}}\left(y_{0}\right)=0$, then

$$
\lambda_{p}=\mathcal{C}_{b}(p)\left(x_{0}, y_{0}, y_{0}\right) \stackrel{(29)}{=} \frac{1}{3} \tilde{\mathcal{C}}_{p}\left(x_{0}\right) \stackrel{(26)}{=} \frac{1}{3}\left(\|\mathcal{C}\|_{p}+\lambda_{p}\right) \Rightarrow \lambda_{p}=\frac{1}{2}\|\mathcal{C}\|_{p}
$$

which is just the upper bound for the eigenvalue $\lambda_{p}$. On the other hand, if

$$
0=\frac{1}{3}-\frac{2}{K^{2}}\left(\frac{2}{3}\right)^{3} \tilde{\mathcal{C}}_{p}^{2}\left(x_{0}\right)
$$

then we have again that

$$
\|\mathcal{C}\|_{p}=\mathcal{C}_{b}(p)\left(x_{0}, x_{0}, x_{0}\right) \stackrel{(29)}{=} \frac{2}{3} \tilde{\mathcal{C}}_{p}\left(x_{0}\right) \stackrel{(26)}{=} \frac{2}{3}\left(\|\mathcal{C}\|_{p}+\lambda_{p}\right) \Rightarrow \lambda_{p}=\frac{1}{2}\|\mathcal{C}\|_{p}
$$

and the lower bound $1-\frac{\|\mathcal{C}\|_{p}^{2}}{4}$ is just equal to the curvature at the point $p$.

Remark 3. Note that the Asanov-type energy functions are singular along the equatorial section defined by the equation $t=0: \ldots$. At the points of the equatorial section, the generatrix of the indicatrix has a corner whose angle is $\beta^{*}=180^{\circ}-\arctan \frac{K}{2} \ldots$ this angle may be regarded as "the parameter of non-Riemannianity"... (cf. Theorem 7; [1]). 
Proposition 1. Suppose that $(V, E)$ is a three-dimensional $\mathcal{C}$-reducible Minkowski space; then for any point $p \in S$

$$
\kappa_{p}=1-\frac{2}{9}\|\mathcal{C}\|_{p}^{2}
$$

Proof. Since the space is $\mathcal{C}$-reducible, the lowered Cartan tensor has the following form:

$$
\mathcal{C}_{b}(p)(x, y, z)=\frac{1}{4}\left(\tilde{\mathcal{C}_{p}}(x) h_{p}(y, z)+\tilde{\mathcal{C}_{p}}(y) h_{p}(x, z)+\tilde{\mathcal{C}_{p}}(z) h_{p}(x, y)\right) .
$$

Using Lemma 1 again, this means that

$$
0=\mathcal{C}_{b}(p)\left(x_{0}, x_{0}, y_{0}\right) \stackrel{(31)}{=} \frac{1}{4} \tilde{\mathcal{C}_{p}}\left(y_{0}\right) \Rightarrow 0=\tilde{\mathcal{C}_{p}}\left(y_{0}\right) .
$$

On the other hand

$$
\lambda_{p}=\mathcal{C}_{b}(p)\left(x_{0}, y_{0}, y_{0}\right) \stackrel{(31)}{=} \frac{1}{4} \tilde{\mathcal{C}_{p}}\left(x_{0}\right) \stackrel{(26)}{=} \frac{1}{4}\left(\|\mathcal{C}\|_{p}+\lambda_{p}\right) \Rightarrow \lambda_{p}=\frac{1}{3}\|\mathcal{C}\|_{p}
$$

and we have (30) by the help of the relation (21).

Finally we are going to reproduce Ji and Shen's result about the curvature of Randers-type indicatrices in case of dimension 3; for the details see [5].

Corollary 1. Suppose that $(V, E)$ is a three-dimensional Minkowski space with a Randers-type energy function; then for any point $p \in S$

$$
0<\kappa_{p} \leq 1 .
$$

Proof. As it is well-known, the norm of the lowered Cartan tensor associated with a Randers-type energy function satisfies the relation

$$
\|\mathcal{C}\|_{p}<\frac{3}{\sqrt{2}}
$$

moreover, it is a $\mathcal{C}$-reducible space and, by Proposition 1 we have the estimation $0<\kappa_{p} \leq 1$ immediately.

\section{REFERENCES}

[1] G. S. Asanov, Finsler cases of GF-spaces, Aequationes Mathematicae, University of Waterloo 49 (1995), 234-251.

[2] G. S. Asanov, Finslerian metric functions over the product $\mathbb{R} \times M$ and their potential applications, Reports on Mathematical Physics, Vol. 41, No.1 (1998), 117-132.

[3] F. Brickell, A theorem on homogeneous functions, J. London Math. Soc., 42 (1967), 325-329.

[4] C. E. Durán, A volume comparaison theorem for Finsler manifolds, Proc. Amer. Moth. Soc., Vol 126, Number 10, October 1998, 3079-3082.

[5] M. Ji and Z. Shen, On strongly convex indicatrices in Minkowski geometry, Cand. Math. Bull., Vol. 45 (2), 2002, 232-246.

[6] R. Schneider, Über die Finslerräume mit $S_{i} j k l=0$, Arch. Math., Vol. XIX, 1968, 656-658.

Institute of Mathematics and Informatics, University of Debrecen, H-4010 Debrecen, P.O.Box 12, Hungary

E-mail address: csvincze@math.klte.hu 